\title{
Units in group rings of crystallographic groups
}

\author{
by
}

\author{
Karel Dekimpe (Kortrijk)
}

\begin{abstract}
In [3], the authors initiated a technique of using affine representations to study the groups of units of integral group rings of crystallographic groups. In this paper, we use this approach for some special classes of crystallographic groups. For a first class of groups we obtain a normal complement for the group inside the group of normalized units. For a second class of groups we show that the Zassenhaus conjectures ZC1 and ZC3 are valid. This generalizes the results known for the infinite dihedral group.
\end{abstract}

1. Crystallographic groups and actions. An $n$-dimensional crystallographic group is a discrete and cocompact subgroup of the group of isometries $\operatorname{Isom}\left(\mathbb{R}^{n}\right)$ of Euclidean $n$-space. These groups are well understood by the three Bieberbach theorems (see [1] and [6]) and the work of Zassenhaus ([7]) who provided a complete algebraic characterization of the crystallographic groups:

A group $\Gamma$ is an $n$-dimensional crystallographic group if and only if it contains a free abelian normal subgroup $A$ of rank $n$, which is of finite index in $\Gamma$ and which is maximal abelian in $\Gamma$.

In other words, a crystallographic group $\Gamma$ fits into a short exact sequence

$$
1 \rightarrow A \cong \mathbb{Z}^{n} \rightarrow \Gamma \rightarrow H \rightarrow 1,
$$

where $H$ is a finite group, called the holonomy group of $\Gamma$. Moreover, the action of $H$ on $A$, induced by conjugation inside $\Gamma$, is a faithful action (since $A$ is maximal abelian in $\Gamma$ ). The maximal abelian normal subgroup $A$ is referred to as the translation subgroup of $\Gamma$. In fact this group $A$ is exactly the group of pure translations, when one realizes $\Gamma$ as a cocompact discrete subgroup of $\operatorname{Isom}\left(\mathbb{R}^{n}\right)$. (Note that by the second Bieberbach theorem such a realization is only unique up to affine conjugation.)

2000 Mathematics Subject Classification: 16S34, $20 \mathrm{C} 07$.

Research supported by a project for bilateral scientific cooperation (Flanders-Poland): BIL01/C-31. 
Although a crystallographic group $H$ can be considered as being a subgroup of the group of isometries of $\mathbb{R}^{n}$, it is often more convenient to regard a crystallographic group as being a group of affine transformations.

Recall that the group $\operatorname{Aff}\left(\mathbb{R}^{n}\right)$ of invertible affine transformations of $\mathbb{R}^{n}$ is the semi-direct product $\mathbb{R}^{n} \rtimes \operatorname{Gl}(n, \mathbb{R})$. An element $(a, A) \in \operatorname{Aff}\left(\mathbb{R}^{n}\right)$ acts on the element $x \in \mathbb{R}^{n}$ by ${ }^{(a, A)} x=a+A x$. The product in $\operatorname{Aff}\left(\mathbb{R}^{n}\right)$ is of course given by $(a, A)(b, B)=(a+A b, A B)$. The affine group $\operatorname{Aff}\left(\mathbb{R}^{n}\right)$ is usually considered to be a subgroup of $\operatorname{Gl}(n+1, \mathbb{R})$ via the representation

$$
\psi: \operatorname{Aff}\left(\mathbb{R}^{n}\right) \rightarrow \operatorname{Gl}(n+1, \mathbb{R}), \quad(a, A) \mapsto\left(\begin{array}{cc}
A & a \\
0 & 1
\end{array}\right) .
$$

We are now ready to explain the type of affine representations we will be considering. We do this in the following (rather well known) lemma.

Lemma 1.1. Let $\Gamma$ be an n-dimensional affine crystallographic group with translation subgroup $A$ and holonomy group $H$. Then there exists a representation $\varphi: \Gamma \rightarrow \operatorname{Aff}\left(\mathbb{R}^{n}\right)$ such that:

(1) $\varphi(\Gamma) \subseteq \mathbb{Q}^{n} \rtimes \mathrm{Gl}(n, \mathbb{Z})$.

(2) $\varphi(A)=\varphi(\Gamma) \cap \mathbb{Q}^{n}=\mathbb{Z}^{n}$.

(3) The induced representation $\psi: H \rightarrow \operatorname{Gl}(n, \mathbb{Z})$ is faithful.

Proof. The crystallographic group $\Gamma$ fits in a short exact sequence $1 \rightarrow$ $\mathbb{Z}^{n} \rightarrow \Gamma \rightarrow H \rightarrow 1$, where we identified $A$ with $\mathbb{Z}^{n}$. Therefore, there exists a normalized 2-cocycle $f: H \times H \rightarrow \mathbb{Z}^{n}$ such that the group $\Gamma$ can be described as the set $\mathbb{Z}^{n} \times H$ with the product given by

$$
\forall z_{1}, z_{2} \in \mathbb{Z}^{n}, \forall h_{1}, h_{2} \in H:\left(z_{1}, h_{1}\right)\left(z_{2}, h_{2}\right)=\left(z_{1}+{ }^{h_{1}} z_{2}+f\left(h_{1}, h_{2}\right), h_{1} h_{2}\right) .
$$

In the formula above, the term ${ }^{h_{1}} z_{2}$ denotes the action of $H$ on $\mathbb{Z}^{n}$ induced by conjugation inside $\Gamma$. We denote this action by $\psi: H \rightarrow \operatorname{Gl}(n, \mathbb{Z})$. (Note that we already mentioned that this action is faithful.) This action can be extended in a unique way to an action of $H$ on $\mathbb{Q}^{n}$, and therefore, we get a new group $\widetilde{\Gamma}$ which has as its underlying set $\mathbb{Q}^{n} \times H$ and where the product is given by

$$
\forall q_{1}, q_{2} \in \mathbb{Q}^{n}, \forall h_{1}, h_{2} \in H:\left(q_{1}, h_{1}\right)\left(q_{2}, h_{2}\right)=\left(q_{1}+{ }^{h_{1}} q_{2}+f\left(h_{1}, h_{2}\right), h_{1} h_{2}\right) .
$$

So, the new group $\widetilde{\Gamma}$ can be seen as an extension of $\mathbb{Q}^{n}$ by $H$ determined by the cocycle $f$, now seen as a 2 -cocycle with coefficients in $\mathbb{Q}^{n}$. However, as $H$ is finite and $\mathbb{Q}^{n}$ is divisible, the second cohomology group $H^{2}\left(H, \mathbb{Q}^{n}\right)$ is 0 . Therefore, there exists a normalized 1-cochain $\lambda: H \rightarrow \mathbb{Q}^{n}$ such that $f=\delta \lambda\left(\right.$ i.e. $\left.f\left(h_{1}, h_{2}\right)={ }^{h_{1}} \lambda\left(h_{2}\right)-\lambda\left(h_{1} h_{2}\right)+\lambda\left(h_{1}\right)\right)$.

It is now easy to check that the map

$$
\varphi: \Gamma=\mathbb{Z}^{n} \times H \rightarrow \mathbb{Q}^{n} \rtimes \operatorname{Gl}(n, \mathbb{Z}), \quad(z, h) \mapsto(z+\lambda(h), \psi(h)),
$$


is a morphism satisfying the three requirements stated above. In matrix notation,

$$
\varphi(z, h)=\left(\begin{array}{cc}
\psi(h) & z+\lambda(h) \\
0 & 1
\end{array}\right)
$$

Definition 1.2. A representation $\varphi: \Gamma \rightarrow \operatorname{Aff}\left(\mathbb{R}^{n}\right)$ of a crystallographic group which satisfies the criteria of the lemma above will be called a suitable representation.

REMARK 1.3. Let $\Gamma$ be a crystallographic group and $\chi: \Gamma \rightarrow \operatorname{Isom}\left(\mathbb{R}^{n}\right)$ an embedding of $\Gamma$ as a discrete cocompact subgroup. Then, for any suitable representation $\varphi: \Gamma \rightarrow \operatorname{Aff}\left(\mathbb{R}^{n}\right)$, there exists an element $\alpha \in \operatorname{Aff}\left(\mathbb{R}^{n}\right)$ such that

$$
\forall \gamma \in \Gamma: \quad \varphi(\gamma)=\alpha \chi(\gamma) \alpha^{-1}
$$

This follows from the second Bieberbach theorem and the fact that any finite subgroup of $\mathrm{Gl}(n, \mathbb{R})$ is conjugate to a subgroup of the orthogonal group $O(n, \mathbb{R})$.

It follows that the affine action of a crystallographic group induced by a suitable representation $\varphi: \Gamma \rightarrow \operatorname{Aff}\left(\mathbb{R}^{n}\right)$ is properly discontinuous and cocompact. Recall that the action of a group $\Gamma$ on $\mathbb{R}^{n}$ is properly discontinuous if and only if for any compact subset $C \subseteq \mathbb{R}^{n}$ the set $\left\{\gamma \in \Gamma \mid{ }^{\gamma} C \cap C \neq \emptyset\right\}$ is finite.

The authors of [3] introduced the idea of extending a given action of a crystallographic group to a ring homomorphism of the corresponding group ring. We follow this idea and therefore we use the usual embedding of $\operatorname{Aff}\left(\mathbb{R}^{n}\right) \subseteq \operatorname{Gl}(n+1, \mathbb{R})$ mentioned above and the inclusion $\operatorname{Gl}(n+1, \mathbb{R}) \subseteq$ $M_{n+1}(\mathbb{R})$ into the ring of $(n+1) \times(n+1)$-matrices. By linearity we can extend any suitable representation $\varphi: \Gamma \rightarrow \operatorname{Aff}\left(\mathbb{R}^{n}\right)$ to a ring homomorphism, which we denote by the same symbol,

$$
\varphi: \mathbb{Z} \Gamma \rightarrow M_{n+1}(\mathbb{R}), \quad \sum_{\gamma \in \Gamma} \alpha_{\gamma} \gamma \mapsto \sum_{\gamma \in \Gamma} \alpha_{\gamma} \varphi(\gamma)
$$

We will use $\mathcal{U}_{1} \mathbb{Z} \Gamma$ to denote the group of normalized units in $\mathbb{Z} \Gamma$, i.e. those units $\sum \alpha_{\gamma} \gamma$ with $\sum \alpha_{\gamma}=1$. Any element $\sum \alpha_{\gamma} \gamma$ of $\mathbb{Z} \Gamma$ is mapped onto a matrix of the form

$$
\left(\begin{array}{ccccc}
a_{1,1} & a_{1,2} & \ldots & a_{1, n} & b_{1} \\
a_{2,1} & a_{2,2} & \ldots & a_{2, n} & b_{2} \\
\vdots & \vdots & \ddots & \vdots & \vdots \\
a_{n, 1} & a_{n, 2} & \ldots & a_{n, n} & b_{n} \\
0 & 0 & \ldots & 0 & \sum \alpha_{\gamma}
\end{array}\right)
$$

where $a_{i, j} \in \mathbb{Z}$ and $b_{i} \in \mathbb{Q}$. 
Moreover, for the elements of $\mathcal{U}_{1} \mathbb{Z} \Gamma$, the image is invertible and the bottom row ends with 1 . Therefore, we find that the image of $\mathcal{U}_{1} \mathbb{Z} \Gamma$ is contained in $\operatorname{Aff}\left(\mathbb{R}^{n}\right)$ (in fact even in $\mathbb{Q}^{n} \rtimes \operatorname{Gl}(n, \mathbb{Z})$ ). With some abuse of notation, we will again use the same symbol $\varphi$ to denote the resulting affine representation $\varphi: \mathcal{U}_{1} \mathbb{Z} \Gamma \rightarrow \operatorname{Aff}\left(\mathbb{R}^{n}\right)$. We will refer to this representation as the affine representation induced by a suitable representation.

REMARK 1.4. The above construction of an affine representation induced by a suitable representation is not exactly the same as the one proposed in [3]. Nevertheless, for both constructions the translation subgroup of $\Gamma$ is mapped onto a discrete group of translations. Therefore, the affine representation obtained in [3] and the one constructed here must be affinely conjugate (this is an easy generalization of the second Bieberbach theorem). Therefore the topological properties of both actions do agree.

It follows that any suitable representation of a crystallographic group induces an affine action of the group $\mathcal{U}_{1} \mathbb{Z} \Gamma$. In most cases, this action will not have any nice topological properties.

ExAmple 1.5. Consider the crystallographic group $\Gamma=\mathbb{Z}^{2} \rtimes S_{3}$, where $S_{3}=\left\langle a, b \mid a^{3}=1, b^{2}=1, b a=a^{-1} b\right\rangle$ acts on $\mathbb{Z}^{2}$ via a map $\psi: S^{3} \rightarrow$ $\mathrm{Gl}(2, \mathbb{Z})$ given by

$$
\psi(a)=\left(\begin{array}{cc}
0 & -1 \\
1 & -1
\end{array}\right), \quad \psi(b)=\left(\begin{array}{cc}
0 & -1 \\
-1 & 0
\end{array}\right) .
$$

If we let $t_{1}=\left(\begin{array}{l}1 \\ 0\end{array}\right)$ and $t_{2}=\left(\begin{array}{l}0 \\ 1\end{array}\right)$ denote the standard free generators for $\mathbb{Z}^{2}$ we obtain a suitable affine representation of $\Gamma$, by

$$
\begin{aligned}
\varphi\left(t_{1}\right)=\left(\begin{array}{lll}
1 & 0 & 1 \\
0 & 1 & 0 \\
0 & 0 & 1
\end{array}\right), & \varphi\left(t_{2}\right)=\left(\begin{array}{lll}
1 & 0 & 0 \\
0 & 1 & 1 \\
0 & 0 & 1
\end{array}\right), \\
\varphi(a)=\left(\begin{array}{ccc}
0 & -1 & 0 \\
1 & -1 & 0 \\
0 & 0 & 1
\end{array}\right), & \varphi(b)=\left(\begin{array}{ccc}
0 & -1 & 0 \\
-1 & 0 & 0 \\
0 & 0 & 1
\end{array}\right) .
\end{aligned}
$$

Let $u=1+(1-b) a(1+b)$. Then $u$ is a bicyclic unit in $\mathcal{U}_{1} \mathbb{Z} S_{3} \subseteq \mathcal{U}_{1} \mathbb{Z} \Gamma$. This $u$ is of infinite order. One checks that

$$
\varphi(u)=\left(\begin{array}{ccc}
4 & -3 & 0 \\
3 & -2 & 0 \\
0 & 0 & 1
\end{array}\right), \quad \text { and so } \quad \varphi\left(u^{k}\right)=\left(\begin{array}{ccc}
1+3 k & -3 k & 0 \\
3 k & 1-3 k & 0 \\
0 & 0 & 1
\end{array}\right)
$$

for all $k \in \mathbb{Z}$. If we now look at the induced affine action of $\mathcal{U}_{1} \mathbb{Z} \Gamma$ on the plane $\mathbb{R}^{2}$, we see that the origin is fixed by this $u^{k}$. On the other hand the distance between the image (under the action of $u^{k}$ ) of any point of the form $\left(\begin{array}{l}x \\ 0\end{array}\right)$, with $x \neq 0$, and the origin goes to infinity as $k$ goes to infinity. This 
implies that the action of $\mathcal{U}_{1} \mathbb{Z} \Gamma$ on $\mathbb{R}^{2}$ cannot be simplicial with respect to any locally finite triangulation of $\mathbb{R}^{2}$.

This fact seems to contradict Corollary 12 of [3]. As this fact is crucially used in the proof of Theorem 15 of that paper, there is also doubt about the exactness of the proof of that theorem. Therefore, we reprove it here. In fact we even prove its generalization.

THEOREM 1.6. Let $\Gamma$ be a crystallographic group, and assume that $\varphi$ : $\mathcal{U}_{1} \mathbb{Z} \Gamma \rightarrow \operatorname{Aff}\left(\mathbb{R}^{n}\right)$ is induced by a suitable representation of $\Gamma$. Then $\mathcal{U}_{1} \mathbb{Z} \Gamma$ $=\Gamma$ if and only if $\mathcal{U}_{1} \mathbb{Z} \Gamma$ acts properly discontinuously on $\mathbb{R}^{n}$.

Proof. If $\mathcal{U}_{1} \mathbb{Z} \Gamma=\Gamma$, then the induced action of $\mathcal{U}_{1} \mathbb{Z} \Gamma$ is exactly the action of $\Gamma$ given by a suitable representation. As mentioned before, this action is properly discontinuous.

Conversely, assume that $\varphi: \mathcal{U}_{1} \mathbb{Z} \Gamma \rightarrow \operatorname{Aff}\left(\mathbb{R}^{n}\right)$ determines a properly discontinuous action. As a point is a compact subset of $\mathbb{R}^{n}$, the stabilizer of each point is finite, and therefore, the kernel of $\varphi$ must also be finite. We claim that the image $\varphi\left(\mathcal{U}_{1} \mathbb{Z} \Gamma\right)$ contains $\varphi(\Gamma) \cong \Gamma$ as a subgroup of finite index. Suppose on the contrary that $\left[\varphi\left(\mathcal{U}_{1} \mathbb{Z} \Gamma\right): \varphi(\Gamma)\right]=\infty$. Then we can choose elements $u_{0}, u_{1}, \ldots, u_{p}, \ldots \in \varphi\left(\mathcal{U}_{1} \mathbb{Z} \Gamma\right)$ for all positive integers $p \in \mathbb{N}$ such that for all $p, q \in \mathbb{N}$,

$$
p \neq q \Rightarrow\left(\forall \gamma_{1}, \gamma_{2} \in \varphi(\Gamma): \gamma_{1} u_{p} \neq \gamma_{2} u_{q}\right)
$$

Now, for any $p \in \mathbb{N}$, we consider the action of $u_{p}$ on the origin 0 . As the group $\mathbb{Z}^{n}$ of integral translations is contained in $\varphi(\Gamma)$, we can find an element $\gamma_{p} \in \mathbb{Z}^{n} \subseteq \varphi(\Gamma)$ such that $\gamma_{p} u_{p} 0 \in[0,1]^{n}$. However, this implies that the set $\left\{\left.\gamma \in \mathcal{U}_{1} \mathbb{Z} \Gamma\right|^{\gamma}[0,1]^{n} \cap[0,1]^{n} \neq \emptyset\right\}$ is infinite, which contradicts the fact that $\mathcal{U}_{1} \mathbb{Z} \Gamma$ acts properly discontinuously.

So, $\left[\varphi\left(\mathcal{U}_{1} \mathbb{Z} \Gamma\right): \varphi(\Gamma)\right]<\infty$ and as the kernel of $\varphi$ is also finite, we have $\left[\mathcal{U}_{1} \mathbb{Z} \Gamma: \Gamma\right]<\infty$. It now follows from the main result of [2] that $\mathcal{U}_{1} \mathbb{Z} \Gamma=\Gamma$, which was to be shown.

To see that the result above is really a generalization of the result of [3], we have to show that any free action of $\mathcal{U}_{1} \mathbb{Z} \Gamma$ is necessarily properly discontinuous. Recall that an action of a group $G$ on a space $X$ is said to be free if ${ }^{g} x=x$ implies $g=1$.

Proposition 1.7. Let $\Gamma$ be a crystallographic group. Assume that $\varphi$ : $\mathcal{U}_{1} \mathbb{Z} \Gamma \rightarrow \operatorname{Aff}\left(\mathbb{R}^{n}\right)$ is induced by a suitable representation of $\Gamma$. If the action of $\mathcal{U}_{1} \mathbb{Z} \Gamma$ determined by $\varphi$ is free, it is also properly discontinuous.

Proof. As the action is free, the map $\varphi$ is injective. The Tits alternative says that $\varphi\left(\mathcal{U}_{1} \mathbb{Z} \Gamma\right)$ is either virtually solvable or contains a non-abelian free subgroup. Suppose that $a, b \in \varphi\left(\mathcal{U}_{1} \mathbb{Z} \Gamma\right)$ generate a free non-abelian subgroup. Then the same holds for any powers $a^{p}$ and $b^{q}$ with $p, q>0$. Now, 
$a, b$ viewed as $(n+1) \times(n+1)$-matrices, belong to $\mathrm{Gl}(n+1, \mathbb{Q})$. Moreover, the characteristic polynomials of both $a$ and $b$ have integral coefficients and unit constant term. It follows from [4] that there exist $p, q>0$ such that $a^{p}$ and $b^{q}$ belong to $\operatorname{Gl}(n+1, \mathbb{Z})$. Regarding these elements as affine transformations again, we find that the image of the origin 0 under the action of $a^{p}$ and $b^{q}$ belongs to $\mathbb{Z}^{n}$. This implies that there exist $z_{1}, z_{2}$ in the translation subgroup of $\Gamma$ such that ${ }^{a^{p}} 0={ }^{z_{1}} 0$ and ${ }^{b^{q}} 0={ }^{z_{2}} 0$, which implies that $a^{p}=z_{1}$ and $b^{q}=z_{2}$. This contradicts the fact that $a^{p}$ and $b^{q}$ generate a free non-abelian group. Therefore $\varphi\left(\mathcal{U}_{1} \mathbb{Z} \Gamma\right)$ is virtually solvable.

Moreover, as $\Gamma$ is a finitely generated subgroup, it follows that there exists an $m \geq 1$ such that $\varphi(\Gamma) \subseteq\left(m^{-1} \mathbb{Z}^{n}\right) \rtimes \operatorname{Gl}(n, \mathbb{Z})$. Therefore also $\varphi\left(\mathcal{U}_{1} \mathbb{Z} \Gamma\right) \subseteq\left(m^{-1} \mathbb{Z}^{n}\right) \rtimes \operatorname{Gl}(n, \mathbb{Z})$. It follows that the virtually solvable group $\varphi\left(\mathcal{U}_{1} \mathbb{Z} \Gamma\right)$ must actually be polycyclic-by-finite. The same reasoning as above shows that for any $u \in \varphi\left(\mathcal{U}_{1} \mathbb{Z} \Gamma\right)$, there exists a $k>0$ such that $u^{k} \in \varphi(\Gamma)$. As we already know that the group $\varphi\left(\mathcal{U}_{1} \mathbb{Z} \Gamma\right)$ is actually polycyclic-by-finite, and not just any (finitely generated) virtually solvable group, we find that $\left[\varphi\left(\mathcal{U}_{1} \mathbb{Z} \Gamma\right): \varphi(\Gamma)\right]<\infty$ (this is in fact the crucial point where the Tits alternative is used). In turn, this implies that the action of $\varphi\left(\mathcal{U}_{1} \mathbb{Z} \Gamma\right) \cong \mathcal{U}_{1} \mathbb{Z} \Gamma$ is properly discontinuous, because the action of $\varphi(\Gamma) \cong \Gamma$ is.

2. Normal complements. Let us recall the very well known theorem of Higman on trivial units (see e.g. [5]):

Theorem 2.1. Let $G$ be a finite group. Then $\mathcal{U} \mathbb{Z} G= \pm G$ if and only if $G$ is abelian of exponent 2, 3, 4 or 6 or $G=E \times Q$, where $Q$ is the quaternion group of order 8 and $E$ is an elementary abelian 2-group.

For crystallographic groups $\Gamma$ having a holonomy group $H$ satisfying the conditions in the above theorem, we can find a normal complement for $\Gamma$ in $\mathcal{U}_{1} \mathbb{Z} \Gamma$.

THEOREM 2.2. Let $\Gamma$ be a crystallographic group with holonomy group $H$, where $H$ is abelian of exponent 2, 3, 4 or 6 or $H=E \times Q$. ( $Q$ is the quaternion group of order 8 and $E$ is an elementary abelian 2-group.) Then there exists a normal complement for $\Gamma$ in $\mathcal{U}_{1} \mathbb{Z} \Gamma$. In fact, if $\varphi: \mathcal{U}_{1} \mathbb{Z} \Gamma \rightarrow$ $\operatorname{Aff}\left(\mathbb{R}^{n}\right)$ is induced by a suitable representation, then the kernel $\operatorname{Ker}(\varphi)$ of $\varphi$ is such a normal complement.

Proof. Choose a set of representatives $h_{1}=1, h_{2}, \ldots, h_{k}$ of the cosets of the translation subgroup $A$, so that any element $\gamma \in \Gamma$ can be uniquely written as $a h_{i}$ for some $i \in\{1, \ldots, k\}$ and $a \in A$. Then any element of $\alpha \in \mathbb{Z} \Gamma$ can be written as a sum

$$
\alpha=\sum_{i=1}^{k} \beta_{i} h_{i} \quad \text { with } \beta_{i} \in \mathbb{Z} A .
$$


The natural projection $p: \Gamma \rightarrow H$ induces a ring morphism

$$
\widetilde{p}: \mathbb{Z} \Gamma \rightarrow \mathbb{Z} H, \quad \sum_{i=1}^{k} \beta_{i} h_{i} \mapsto \sum_{i=1}^{k} \varepsilon\left(\beta_{i}\right) p\left(h_{i}\right),
$$

where $\varepsilon$ denotes the augmentation map as usual.

Now, assume that $u \in \mathcal{U}_{1} \mathbb{Z} \Gamma$; then $\widetilde{p}(u) \in \mathcal{U}_{1} \mathbb{Z} H$, and therefore, $\widetilde{p}(u)=$ $p\left(h_{i_{0}}\right)$ for some $i_{0} \in\{1, \ldots, k\}$ (by Theorem 2.1). It follows that $\varepsilon\left(\beta_{j}\right)=0$ if $j \neq i_{0}$ and $\varepsilon\left(\beta_{i_{0}}\right)=1$. Note that for any element of $a \in \mathbb{Z} A$, we have

$$
\varphi(a)=\left(\begin{array}{ccccc}
\varepsilon(a) & 0 & \ldots & 0 & a_{1} \\
0 & \varepsilon(a) & \ldots & 0 & a_{2} \\
\vdots & \vdots & \ddots & \vdots & \vdots \\
0 & 0 & \ldots & \varepsilon(a) & a_{n} \\
0 & 0 & \ldots & 0 & \varepsilon(a)
\end{array}\right)
$$

for some $a_{1}, \ldots, a_{n} \in \mathbb{Z}$.

It follows that there exist $\mathbf{a}_{i}, \mathbf{b}_{i} \in \mathbb{Z}^{n}$ such that

$$
\begin{aligned}
& \varphi(u)=\sum_{i=1}^{k} \varphi\left(\beta_{i}\right) \varphi\left(h_{i}\right) \\
& =\left(\begin{array}{cc}
\mathbf{O} & \mathbf{a}_{1} \\
0 & 0
\end{array}\right)\left(\begin{array}{cc}
\psi\left(p\left(h_{1}\right)\right) & \mathbf{b}_{1} \\
0 & 1
\end{array}\right)+\left(\begin{array}{cc}
\mathbf{O} & \mathbf{a}_{2} \\
0 & 0
\end{array}\right)\left(\begin{array}{cc}
\psi\left(p\left(h_{2}\right)\right) & \mathbf{b}_{2} \\
0 & 1
\end{array}\right)+\ldots \\
& +\left(\begin{array}{cc}
\mathbf{I} & \mathbf{a}_{i_{0}} \\
0 & 1
\end{array}\right)\left(\begin{array}{cc}
\psi\left(p\left(h_{i_{0}}\right)\right) & \mathbf{b}_{i_{0}} \\
0 & 1
\end{array}\right)+\ldots+\left(\begin{array}{cc}
\mathbf{O} & \mathbf{a}_{k} \\
0 & 0
\end{array}\right)\left(\begin{array}{cc}
\psi\left(p\left(h_{k}\right)\right) & \mathbf{b}_{k} \\
0 & 1
\end{array}\right) \\
& =\left(\begin{array}{cc}
\psi\left(p\left(h_{i_{0}}\right)\right) & \mathbf{a}_{1}+\mathbf{a}_{2}+\ldots+\mathbf{a}_{k}+\mathbf{b}_{i_{0}} \\
0 & 1
\end{array}\right) \\
& =\left(\begin{array}{cc}
\mathbf{I} & \mathbf{a}_{1}+\mathbf{a}_{2}+\ldots+\mathbf{a}_{k} \\
0 & 1
\end{array}\right)\left(\begin{array}{cc}
\psi\left(p\left(h_{i_{0}}\right)\right) & \mathbf{b}_{i_{0}} \\
0 & 1
\end{array}\right) \\
& =\varphi\left(a h_{i_{0}}\right) \quad \text { for some } a \in A \text {. }
\end{aligned}
$$

In the computations above, $\mathbf{O}$ (resp. I) stands for the $n \times n$ zero matrix (resp. identity matrix).

It follows that $\varphi\left(\mathcal{U}_{1} \mathbb{Z} \Gamma\right)=\varphi(\Gamma)$ and hence we have a split short exact sequence

$$
1 \rightarrow \operatorname{Ker}(\varphi) \rightarrow \mathcal{U}_{1} \mathbb{Z} \Gamma \rightarrow \varphi(\Gamma) \rightarrow 1,
$$

where the splitting is of course given by $s: \varphi(\Gamma) \rightarrow \mathcal{U}_{1} \mathbb{Z} \Gamma, \varphi(\gamma) \mapsto \gamma$. This shows that $\operatorname{Ker}(\varphi)$ is a normal complement to $\Gamma$ inside $\mathcal{U}_{1} \mathbb{Z} \Gamma$.

3. Crystallographic groups with holonomy $\mathbb{Z}_{2}$. In [5, Section 46], the unit group of the infinite dihedral subgroup is studied. This is a 1dimensional crystallographic group with holonomy group $\mathbb{Z}_{2}$. In this section we will conduct an analogous study for all crystallographic groups with 
holonomy $\mathbb{Z}_{2}$. In fact, this section follows very closely the proof of Theorem (46.3) (which only treats the infinite dihedral group case) in [5].

We begin by showing that for crystallographic groups with a holonomy of order 2, the normal complement obtained in the previous section is torsionfree.

THEOREM 3.1. Let $\Gamma$ be an $n$-dimensional crystallographic group with a holonomy group $H \cong \mathbb{Z}_{2}$. Let $\operatorname{Ker}(\varphi)$ be the kernel of the morphism $\varphi$ : $\mathcal{U}_{1} \mathbb{Z} \Gamma \rightarrow \operatorname{Aff}\left(\mathbb{R}^{n}\right)$ which is induced by a suitable affine representation of $\Gamma$. Then $\operatorname{Ker}(\varphi)$ is torsion-free and is a normal complement to $\Gamma$ inside $\mathcal{U}_{1} \mathbb{Z} \Gamma$.

Proof. The fact that $\operatorname{Ker}(\varphi)$ is a normal complement to $\Gamma$ is the content of Theorem 2.2 .

If $\Gamma$ is torsion-free, then $\mathcal{U}_{1} \mathbb{Z} \Gamma$ is also torsion-free, and then obviously so is $\operatorname{Ker}(\varphi)$. So for the rest of this proof, we can assume that $\Gamma$ is not torsion-free. In this case $\Gamma$ is a semi-direct product

$$
\Gamma=A \rtimes \mathbb{Z}_{2},
$$

where $A \cong \mathbb{Z}^{n}$ is the translation subgroup of $\Gamma$. We denote the generator of $\mathbb{Z}_{2}$ by $b$, and choose a set of generators $t_{1}, \ldots, t_{n}$ of $A$. Then any element $\alpha$ of $\mathbb{Z} \Gamma$ can be written as

$$
\alpha=f\left(t_{1}, \ldots, t_{n}\right)+g\left(t_{1}, \ldots, t_{n}\right) b
$$

for some Laurent polynomials

$$
f\left(t_{1}, \ldots, t_{n}\right), g\left(t_{1}, \ldots, t_{n}\right) \in \mathbb{Z}\left[t_{1}, t_{1}^{-1}, \ldots, t_{n}, t_{n}^{-1}\right] .
$$

For brevity, we will write $f(t)$ when we mean a Laurent polynomial of the form $f\left(t_{1}, \ldots, t_{n}\right)$. Now, assume that $u=f(t)+g(t) b \in \mathcal{U}_{1} \mathbb{Z} \Gamma$ is a torsion unit. Then $g(t) \neq 0$. (Recall that $\mathcal{U}_{1} \mathbb{Z} A=A$, since $A$ is an ordered group.) As $\Gamma$ only has torsion of order 2 , the order of the element $u$ must be 2 as well (see [5, Theorem (45.11)]).

The element $b$ induces an involution * on $\mathbb{Z}\left[t_{1}, t_{1}^{-1}, \ldots, t_{n}, t_{n}^{-1}\right]$ by defining

$$
\left(t_{1}^{k_{1}} t_{2}^{k_{2}} \ldots t_{n}^{k_{n}}\right)^{*}=b\left(t_{1}^{k_{1}} t_{2}^{k_{2}} \ldots t_{n}^{k_{n}}\right) b^{-1}
$$

for each monomial $t_{1}^{k_{1}} t_{2}^{k_{2}} \ldots t_{n}^{k_{n}}\left(k_{1}, k_{2}, \ldots, k_{n} \in \mathbb{Z}\right)$ and extending this linearly. Of course, on the right hand side of (1), we interpret the monomial $t_{1}^{k_{1}} t_{2}^{k_{2}} \ldots t_{n}^{k_{n}}$ as being an element of $A$ and we compute the conjugation inside $\Gamma$.

Using this involution, we can now compute

$$
\begin{aligned}
1 & =u^{2}=(f(t)+g(t) b)^{2} \\
& =f^{2}(t)+g(t) g^{*}(t)+\left(f(t) g(t)+f^{*}(t) g(t)\right) b .
\end{aligned}
$$

This implies that $\left(f(t)+f^{*}(t)\right) g(t)=0$. As $g(t) \neq 0$ and $\mathbb{Z}\left[t_{1}, t_{1}^{-1}, \ldots, t_{n}, t_{n}^{-1}\right]$ 
is an integral domain, we find that

$$
f(t)+f^{*}(t)=0 \quad \text { or } \quad f(t)=-f^{*}(t) .
$$

However, as $\varepsilon(f(t))=\varepsilon\left(f^{*}(t)\right)$, this implies that $\varepsilon(f(t))=0$ and hence $\varepsilon(g(t))=1$. This implies that $\varphi(u)=\varphi(f(t)+g(t) b)=\varphi(a b) \neq 1$ (for some $a \in A)$, showing that $u \notin \operatorname{Ker}(\varphi)$, which shows that $\operatorname{Ker}(\varphi)$ is torsion-free.

Corollary 3.2. Let $\Gamma$ be a crystallographic group with holonomy group $\mathbb{Z}_{2}$. Then any non-trivial finite subgroup of $\mathcal{U}_{1} \mathbb{Z} \Gamma$ is isomorphic to $\mathbb{Z}_{2}$.

Proof. As $\operatorname{Ker}(\varphi)$ is torsion-free and $\mathcal{U}_{1} \mathbb{Z} \Gamma=(\operatorname{Ker}(\varphi)) \Gamma$, any finite subgroup is isomorphic to a finite subgroup of $\Gamma$. Moreover, as $A$ is torsion-free, any finite subgroup of $\Gamma$ is isomorphic to a finite subgroup of the holonomy group $\Gamma / A \cong \mathbb{Z}_{2}$. Therefore, any non-trivial finite subgroup is mapped isomorphically onto $\mathbb{Z}_{2}$.

This corollary, together with the following theorem, proves that the Zassenhaus conjectures ZC1 and ZC3 hold for the class of groups under investigation.

THEOREM 3.3 (ZC1). Let $\Gamma$ be a crystallographic group with holonomy group $\mathbb{Z}_{2}$. Then any torsion unit $u \in \mathcal{U}_{1} \mathbb{Z} \Gamma$ is conjugate inside $\mathbb{Q} \Gamma$ to an element of $\Gamma$.

Proof. We recall the proof and notations of Theorem 3.1, where we showed that a torsion element $u \in \mathcal{U}_{1} \mathbb{Z} \Gamma$ is of the form $u=f(t)+g(t) b$, is of order 2 and satisfies $\varepsilon(f(t))=0$ and $\varepsilon(g(t))=1$. By (2) and (3) we find that $1=f^{2}(t)+g(t) g^{*}(t)$, and so

$$
g(t) g^{*}(t)=1-f^{2}(t)=(1+f(t))(1-f(t))=(1+f(t))(1+f(t))^{*} .
$$

As $\mathbb{Z}\left[t_{1}, t_{1}^{-1}, \ldots, t_{n}, t_{n}^{-1}\right]$ is a unique factorization domain, the relation

$$
g(t) g^{*}(t)=(1+f(t))(1+f(t))^{*}
$$

implies the existence of two elements $p(t), q(t) \in \mathbb{Z}\left[t_{1}, t_{1}^{-1}, \ldots, t_{n}, t_{n}^{-1}\right]$ and a monomial $\mathbf{t}=t_{1}^{k_{1}} \ldots t_{n}^{k_{n}}$ such that

$$
1+f(t)=p(t) q(t), \quad g(t)= \pm \mathbf{t} p(t) q^{*}(t) .
$$

In fact, as $\varepsilon(1+f(t))=1=\varepsilon(g(t))$, we must actually have $g(t)=\mathbf{t} p(t) q^{*}(t)$. The relation $g(t) g^{*}(t)=(1+f(t))(1+f(t))^{*}$ implies that $\mathbf{t t}^{*}=1$ (i.e. $\left.\mathbf{t}^{*}=\mathbf{t}^{-1}\right)$. Finally, $f(t)+f^{*}(t)=0$ shows that $p(t) q(t)+p^{*}(t) q^{*}(t)=2$.

Let

$$
\alpha=\frac{p^{*}(t)+q(t)}{2}+\frac{q^{*}(t)-p(t)}{2} \mathbf{t} b .
$$

Then $\alpha \in \mathcal{U} \mathbb{Q} \Gamma$, with

$$
\alpha^{-1}=\frac{p(t)+q^{*}(t)}{2}+\frac{p(t)-q^{*}(t)}{2} \mathbf{t} b .
$$


Moreover,

$$
\begin{aligned}
2 \alpha u= & \left(\left(p^{*}(t)+q(t)\right)+\left(q^{*}(t)-p(t)\right) \mathbf{t} b\right)\left(p(t) q(t)-1+p(t) q^{*}(t) \mathbf{t} b\right) \\
= & -p^{*}(t)+p(t) q(t)^{2}-q(t)+p^{*}(t) q^{*}(t) q(t) \\
& +\left[q(t) p(t) q^{*}(t)+p(t)+q^{*}(t)^{2} p^{*}(t)-q^{*}(t)\right] \mathbf{t} b \\
= & -p^{*}(t)+p(t) q(t)^{2}-q(t)+(2-p(t) q(t)) q(t) \\
& +\left[q(t) p(t) q^{*}(t)+(2-p(t) q(t)) q^{*}(t)-q^{*}(t)+p(t)\right] \mathbf{t} b \\
= & 2 \mathbf{t} b \alpha
\end{aligned}
$$

which shows that $\alpha u \alpha^{-1}=\mathbf{t} b \in \Gamma$.

Corollary 3.4 (ZC3). Let $\Gamma$ be a crystallographic group with holonomy group $\mathbb{Z}_{2}$. If $F$ is a finite subgroup of $\mathcal{U}_{1} \mathbb{Z} \Gamma$, then $F$ is conjugate by an element of $\mathbb{Q} \Gamma$ to a subgroup of $\Gamma$.

Proof. This is an immediate consequence of Corollary 3.2 and Theorem 3.3.

Acknowledgements. The author expresses his gratitude towards the Mathematics Department of the University of Warsaw, for their hospitality during his stay in April 2002. It is during this stay that he got acquainted with the work of Marciniak and Sehgal.

\section{References}

[1] L. S. Charlap, Bieberbach Groups and Flat Manifolds, Universitext, Springer, New York, 1986.

[2] D. R. Farkas and P. A. Linnell, Trivial units in group rings, Canad. Math. Bull. 43 (2000), 60-62.

[3] Z. S. Marciniak and S. K. Sehgal, Units in group rings and geometry, in: Methods in Ring theory (Levico Terme, 1997), Lecture Notes in Pure and Appl. Math. 198, Marcel Dekker, New York, 1998, 185-198.

[4] H. L. Porteous, Anosov diffeomorphisms of flat manifolds, Topology 11 (1972), 307-315.

[5] S. K. Sehgal, Units in Integral Group Rings, Pitman Monogr. Surveys Pure Appl. Math. 69, Longman Sci. Tech., Harlow, 1993.

[6] J. A. Wolf, Spaces of Constant Curvature, Publish or Perish, Berkeley, 1977.

[7] H. Zassenhaus, Über einen Algorithmus zur Bestimmung der Raumgruppen, Comment. Math. Helv. 21 (1948), 117-141.

Katholieke Universiteit Leuven

Campus Kortrijk

B-8500 Kortrijk, Belgium

E-mail: Karel.Dekimpe@kulak.ac.be

Received 27 May 2003;

in revised form 24 October 2003 